\title{
A 10-state model for an AMC scheme with repetition coding in mobile wireless networks
}

\author{
Nguyen Quoc-Tuan ${ }^{1}$, Dinh-Thong Nguyen ${ }^{2^{*}}$ and Lam Sinh Cong ${ }^{1}$
}

\begin{abstract}
In modern broadband wireless access systems such as mobile worldwide interoperability for microwave access (WiMAX) and others, repetition coding is recommended for the lowest modulation level, in addition to the mandatory concatenated Reed-Solomon and convolutional code data coding, to protect vital control information from deep fades. This paper considers repetition coding as a time-diversity technique using maximum ratio combining (MRC) and proposes techniques to define and to calculate the repetition coding gain $G_{r}$ and its effect on bit error rate (BER) under the two fading conditions: correlated lognormal shadowing and composite Rayleigh-lognormal fading also known as Suzuki fading. A variable-rate, variable-power 10-state finite-state Markov channel (FSMC) model is proposed for the implementation of the adaptive modulation and coding (AMC) scheme in mobile WiMAX to maximize its spectral efficiency under constant power constraints in the two fading mechanisms. Apart from the proposed FSMC model, the paper also presents two other significant contributions: one is an innovative technique for accurate matching of moment generating functions, necessary for the estimation of the probability density function of the combiner's output signal-to-noise ratio, and the other is efficient and fast expressions using Gauss-Hermite quadrature approximation for the calculation of BER of QPSK signal using MRC diversity reception.
\end{abstract}

Keywords: Lognormal fading; Suzuki fading; Gauss-Hermite polynomial; Moment generating function; WiMAX; Adaptive modulation and coding; Repetition coding; Finite-state Markov channel model

\section{Introduction}

In modern wireless communication networks such as $3 \mathrm{G}$ long-term evolution and WiMAX, modulation and coding are adapted to the fading condition of the channel, typically to the received signal-to-noise ratio (SNR) fed back to the base station by the subscriber station. This adaptive modulation and coding (AMC) scheme is usually designed to maximize the system average spectral efficiency over the whole fading range while maintaining a fixed given target bit error rate (BER). Adaptive transmission is usually performed by adjusting the transmit power level, the modulation level, the coding rate, or a combination of these parameters, in order to maintain a constant ratio of bit energy-to-additive white Gaussian noise $\left(E_{\mathrm{b}} / N_{0}\right)$. For a given target BER, the system can achieve high average spectral efficiency by transmitting at high rates for high channel SNR and at lower rates for poorer channel SNR.

\footnotetext{
* Correspondence: dinh-thong.nguyen@uts.edu.au

2University of Technology, Sydney, Sydney, New South Wales, Australia Full list of author information is available at the end of the article
}

For reasons of inherently high spectral efficiency and ease of implementation, modulation as well as coding in modern mobile wireless networks are restricted to a finite set, e.g., to square QAM constellation size of $M=\{4,16$, $64,256\}$, to coding rates of $R=\{1 / 2,2 / 3,3 / 4,5 / 6\}$. In the IEEE 802.16e standard for mobile WiMAX [1], repetition coding $(\mathrm{RC})$ with the number of repetition times $x=\{2,4,6\}$ is also applied to QPSK for diversity gain in order to protect vital control information during deep fading. Thus, the scheme forms a discrete set of combined modulation and coding specified by the corresponding standard. By partitioning the range of the received SNR into a finite number of intervals, a finitestate Markov channel (FSMC) model can be constructed for the implementation of the AMC scheme in a Rayleigh fading wireless channel [2-6]. Corresponding analysis in a lognormal shadow fading and in Rayleighlognormal composite fading environments is far sparser because of the complexity of the underlining lognormal probability theories [7-9], especially when correlation

\section{实 Springer}


between diversity channels is taken into consideration. Moreover, the physics of shadowing and its lognormality statistical property are not well understood [10]. In a widely quoted paper [11], Suzuki presents a simple physical model for radio propagation suitable for typical mobile radio propagation between the base station and a mobile receiver in urban areas, in which the probability density function for the fading follows a composite Rayleigh-lognormal distribution.

In FSMC theory, the partition of SNR into state intervals or regions can be arbitrary; e.g., in [2] the equal steady-state probability method is used to determine the SNR thresholds of the states, while in [3] the equal average state duration is assumed. However, in practice the system's physical parameters are usually standardized and our proposed FSMC model for the fading wireless channel is 'tailored' to conform to the relevant physical standard. Thus, while FSMC is a model of the fading channel, the proposed model in our paper is also a function of the particular modulation and coding schemes used by the physical system. In order not to 'abuse' the basic definition of a Markov process, the necessary assumption in our model is that the channel fading is slow enough so that the SNR remains within one SNR region over several resource allocation unit times, and thus the Markov process can only transit to the same region or to the two adjacent regions. Since the IEEE 802.16e standard [1] gives only a finite number of profile AMC schemes, it is logical to use these profile AMC schemes as the finite states of the FSMC model for mobile WiMAX as shown in Table 1 .

Current research in the literature on FSMC modeling of fading wireless channels has also not addressed adequately the effects of data coding on BER. The concatenated Reed-Solomon and convolutional code (RS-CC) is mandatory in most wireless systems, and others such as convolutional turbo code, block turbo code, and low-density

\section{Table 1 A 10-state FSMC model for mobile WiMAX.}

\begin{tabular}{lccc}
\hline Modulation & $\begin{array}{c}\text { Coding rate, } \\
\text { repetition }\end{array}$ & $\begin{array}{c}\text { Spectral efficiency } \boldsymbol{C}_{\boldsymbol{j}} \\
\text { (bps/Hz) }\end{array}$ & $\begin{array}{c}\text { State } \\
\boldsymbol{s}_{\boldsymbol{j}}\end{array}$ \\
\hline QPSK & $\mathrm{R} 1 / 2,6 \times$ & 0.17 & 1 \\
$\mathrm{R} 1 / 2,4 \times$ & 0.25 & 2 \\
$\mathrm{R} 1 / 2,2 \times$ & 0.50 & 3 \\
$\mathrm{R} 1 / 2$ & 1.00 & 4 \\
$\mathrm{R} 3 / 4$ & 1.50 & 5 \\
16-QAM & $\mathrm{R} 1 / 2$ & 2.00 & 6 \\
$\mathrm{R} 3 / 4$ & 3.00 & 7 \\
$\mathrm{R} 1 / 2$ & 3.00 & 8 \\
$\mathrm{R} 2 / 3$ & 4.00 & 9 \\
$\mathrm{R} 3 / 4$ & 4.50 & 10 \\
\hline
\end{tabular}

parity-check code are optional alternatives. Since data coding results in an effective power gain, corresponding convolutional coding gain $\left(G_{\mathrm{c}}\right)$ and repetition coding gain $\left(G_{\mathrm{r}}\right)$ must be applied to obtain an effective SNR for the implementation of the AMC scheme in mobile wireless networks. The effect of coding gain of trellis code on power adaptation in a four-state $M$-QAM signal has been addressed in [4]. In repetition coding in an OFDMA system, the same data symbol is transmitted on several contiguous slots so that if the information on one of those slots is corrupted, the information on the other slots will be received correctly by a maximum ratio combining (MRC) receiver. The obvious downside of repetition coding is that it decreases the spectral efficiency and this is why the most robust modulation BPSK is not used with repetition coding.

In this paper, we present a 10-state FSMC model for the AMC scheme in mobile WiMAX, taking into account also the repetition coding gain in two different fading scenarios: correlated lognormal fading and composite Rayleighlognormal fading, also known as Suzuki fading. Because the main theme of our paper is the effect of repetition coding on the proposed 10-state FSMC model for AMC control, but not on channel fading models, we will restrict ourselves, for simplicity and brevity, to the Rayleighdistributed channel (voltage) gain and the corresponding exponentially distributed channel (power) gain rather than dealing with their respective generic distributions, i.e., Nakagami- $m$ distribution and gamma- $k$ distribution, respectively. One of the significant findings in this paper is that the channel fading correlation, while significantly degrading the BER performance, practically does not affect the proposed variable power control algorithm and its resulting 10-state FSMC model for mobile WiMAX. This is because repetition coding is applied only to the first three states, but the total power in these states is too small to affect the overall variable power control scheme.

To the best of our knowledge, the performance of repetition coding has not been studied before, partly because the flexible allocation of the OFDMA slots in the timefrequency domain and the nature of the diversity channels involved in the transmission of the repetition slots are not well understood. This will be discussed in Section 2.2. The approach proposed in the paper can be generalized to design power control algorithm for other wireless communication systems using AMC under fading conditions.

In this paper we also show that many complicated expressions for BER involving integrations and double integrations of lognormal and lognormal-related composite functions can be efficiently and accurately approximated in closed form using Gauss-Hermite quadrature polynomials.

There are three main contributions from this paper. The first is an innovative technique for accurate matching of two moment generating functions using 
the power conservation principle: one is the moment generating function (MGF) of the sum of SNRs at the output of the MRC combiner and the other is of an accurate estimate of this sum. Current MGF matching techniques to date, e.g. [9], are seriously power 'lossy' and rather unreliable. The second is the most computationally simple closed-form expression to date for an accurate approximation of BER of QPSK signals using MRC diversity reception operating in correlated lognormal (expression (23)) and composite Rayleigh-lognormal (expression (30)) fading environments. The third is the definition of the repetition coding gain $G_{\mathrm{r}}$ and its incorporation into the design of the transmit power control policy of a 10-state FSMC model for the AMC scheme in mobile WiMAX using repetition coding for QPSK signal. The work in this paper is particularly relevant to the interests of both designers and researchers of broadband wireless access networks.

The rest of the paper is organized as follows. In Section 2, we briefly present the time-diversity model for the repetition coding in an OFDMA system and the bound on BER of the rectangular $M$-QAM signal which serves as the foundation of the transmit power control algorithm originally proposed in $[4,5]$. Section 3 presents an analysis of the effect on BER of QPSK signals from the use of repetition coding under the two fading conditions: correlated lognormal fading and composite Rayleigh-lognormal fading. In this section, we also define and calculate the $\mathrm{RC}$ gain for the two fading conditions. In this section, an innovative technique is presented for accurate matching of two MGFs. In Section 4, we present the steps in the algorithm leading to a 10-state FSMC model for implementing the AMC scheme in mobile WiMAX operating in the mentioned fading environments. Finally, a conclusion is presented in Section 5.

\section{Signal model, repetition diversity channel model, and bound on bit error rate}

\subsection{Signal model}

In this paper the signal-to-noise ratio, $\gamma$, plays a major role in channel characterization and performance evaluation and it can be defined from the signal model:

$$
r(t)=h s(t)+n(t),
$$

where $r(t), s(t)$, and $n(t)$ are receive signal, transmit signal, and channel noise, respectively; $h$ is the amplitude channel gain, assumed to be constant over the transmission time of an orthogonal frequency division multiplex (OFDM) symbol block, thus preserving the orthogonality between subcarriers; $n(t)$ is modeled as a zero-mean additive white Gaussian noise (AWGN) process with one-sided power spectral density $N_{0}$. The received SNR is then

$$
\gamma=\frac{\left|h^{2}\right| E_{\mathrm{s}}}{N_{0}}
$$

where the signal energy is $E_{\mathrm{s}}=E\left[s^{2}(t)\right]$. If the energy is that of 1 bit, then we denote $\gamma_{\mathrm{b}}$ as the SNR per bit of transmitted information.

In this paper we use the term power gain $p=|h|^{2}$ and signal-to-noise ratio $\gamma$ interchangeably where it is appropriate. Since per bit SNR is $\gamma_{\mathrm{b}}=|h|^{2} \times E_{\mathrm{b}} / N_{0}$ and to avoid dealing with the distance dependency, we normalize the average channel power gain $E\left[|h|^{2}\right]=1$, thus making the average received SNR per bit per channel $\bar{\gamma}_{\mathrm{b}}=E_{\mathrm{b}} / N_{0}$.

\subsection{Diversity channel model for repetition coding in OFDMA systems}

In the AMC zone of an OFDMA frame in IEEE802.16e [1], subchannels are formed from grouping of adjacent subcarriers. Adjacent subcarrier allocation results in subchannels which are suitable for frequency non-selective and slowly fading channels, e.g., lognormal shadowing. In an OFDMA system, the basic unit of resource allocation in the 2-D frequency-time grid is the slot being 1 subchannel in frequency by 1 , two or three OFDM symbols in time. More slots can be concatenated to accommodate larger forward error correction (FEC) encoded data blocks. Since repetition coding repeats the same encoded data block in different contiguous slots in the AMC zone, it can be assumed that the MRC gain from combining repeating signals is predominantly via microdiversity reception in which all repetition subchannels experience the same shadowing having $N\left(\mu_{Z}, \sigma_{Z}^{2}\right)$ distribution. The time separation, hence the correlation coefficient between any two diversity subchannels, depends on the size of the FEC-encoded data blocks to be repeated as well as the speed of the mobile receiver.

\subsection{Bound on BER in rectangular M-QAM}

At high SNR, the symbol-error-rate for rectangular $M$-QAM in AWGN with $M=2^{k}$, when $k$ is even, is approximated as [12], p. 280

$$
\operatorname{SER}_{\mathrm{AWGN}, M-\mathrm{QAM}} \approx 4\left(1-\frac{1}{\sqrt{M}}\right) Q\left(\sqrt{\frac{3}{M-1} \gamma_{\mathrm{s}}}\right),
$$

in which is the average SNR per symbol per channel (without combining) and for equiprobable orthogonal signals the corresponding bit error rate is [12], p. 262

$$
\mathrm{BER}_{\mathrm{AWGN}, M-\mathrm{QAM}}=\frac{M}{2(M-1)} \operatorname{SER}_{\mathrm{AWGM}, M-\mathrm{QAM}}(\gamma) .
$$

By using the asymptotic expansion of the function $Q$ $(x)$ in (3), an upper bound for BER for a given value of SNR is given in $[4,6]$

$$
\operatorname{BER}_{\mathrm{AWGN}, M-\mathrm{QAM}}(\gamma) \leq K_{\mathrm{B}}(M) \exp \left(-\frac{1.5 \gamma}{M-1}\right)
$$


in which the bound constant $K_{\mathrm{B}}(M)$ is fixed at 0.2 in [4] and is given as a function of $M$ in [6] as

$$
K_{\mathrm{B}}(M)=0.266\left(\frac{M}{M-1}\right)\left(1-\frac{1}{\sqrt{M}}\right) .
$$

It is obvious that for $M>4$, the upper bound for BER in (5) given by [4] is very tight, and this bound or its power adaptation version in (54) provides the basis for the transmit power control algorithm in [4] and [5].

\section{Effect of repetition coding on BER and effective repetition coding gain}

\subsection{Repetition coding for QPSK in WiMAX}

In this paper, we define repetition coding gain simply as the ratio of the SNR without repetition coding to the SNR with repetition coding for a given target BER. Thus, an improvement in BER is equivalent to a saving in signaling power required to combat deep fades in order to maintain the given target BER. Since in the AMC scheme in mobile WiMAX, and repetition coding of 6,4 , and 2 times is recommended only for rate $1 / 2$ QPSK modulation and coding (see Table 1), it is important that we first derive accurate closed-form formulas for BER of QPSK signals from an MRC combiner and the corresponding RC gain when the wireless system operates in lognormal shadowing and in composite Rayleigh-lognormal fading environments. This is one of the significant contributions from our paper.

\subsection{Correlated lognormal fading channels only}

3.2.1 Power sum of correlated lognormal random variables

A signal subjected to shadowing, also known as slow fading, is usually modeled as a lognormally distributed random variable. Its SNR is modeled as $\gamma=10^{0.1 Z}=\exp (Z / \xi)$ with $Z$ in decibels being normally distributed, i.e., $Z \sim N$ $\left(\mu_{Z}, \sigma_{Z}^{2}\right)$. The probability density function of $\gamma$ is

$$
f_{\text {lognormal }}(\gamma)=\frac{1}{\gamma} \frac{\xi}{\sigma_{z} \sqrt{2 \pi}} \exp \left(-\frac{\left(10 \log _{10} \gamma-\mu_{z}\right)^{2}}{2 \sigma_{z}^{2}}\right)
$$

in which $\xi=10 / \log 10$ is the conversion constant between $d B$ and net and is in linear unit. The average SNR is

$$
\bar{\gamma}_{\mathrm{Ln}}=\exp \left[\frac{\mu_{z}}{\xi}+\frac{1}{2}\left(\frac{\sigma_{z}}{\xi}\right)^{2}\right] .
$$

The effect of maximum ratio combining is to add up the powers of the received signals to be combined. The resulting SNR from $N$ repetitions is

$$
\begin{aligned}
\gamma_{N} & =\sum_{i=1}^{N} \gamma_{i} \\
& =\sum_{i=1}^{N} 10^{0.1 Z_{i}} \text { with } Z_{i} \sim N\left(\mu_{Z i}, \sigma_{Z i}{ }^{2}\right)
\end{aligned}
$$

A closed-form expression for the probability density function (PDF) of the power sum of lognormal random variables (RVs) in (9) is not available, but a number of approximations in computationally efficient closed forms are currently available. These include the Pearson Type IV approximation in $[7,8]$ and those found from the MGF matching technique in [9]. In our paper, we adopt the latter approach because it is elegant and simple and it results in a PDF expression being suitable for the use of Gauss-Hermite expansion to approximate the BER in a closed form.

Consider the $N$ correlated lognormal RV vector $\gamma=\left\{\gamma_{i}\right\}$, $i=1,2, . ., N$, and their corresponding Gaussian RV vector $z=\left\{z_{i}\right\}$, having the joint distribution

$$
f_{z}(\boldsymbol{z})=\frac{1}{(2 \pi)^{N / 2}\left|\boldsymbol{C}_{\boldsymbol{z}}\right|^{1 / 2}} \exp \left(-\frac{(\boldsymbol{z}-\boldsymbol{\mu})^{T} C_{z}^{-1}(\boldsymbol{z}-\boldsymbol{\mu})}{2}\right),
$$

where $\boldsymbol{\mu}$ is the mean vector of $\mathbf{z}$ and $\mathbf{C}_{\mathbf{Z}}$ is the covariance matrix of $\mathbf{z}$.

After equating $f_{\mathbf{Y}}(\boldsymbol{\gamma}) d \boldsymbol{\gamma}=f_{\mathbf{z}}(\mathbf{z}) d \mathbf{z}$, the MGF of the combined SNR is obtained as

$$
\begin{aligned}
M_{\gamma_{N}}(s)= & \int_{-\infty}^{\infty} \frac{1}{(2 \pi)^{N / 2}\left|\boldsymbol{C}_{\boldsymbol{z}}\right|^{1 / 2} \Pi_{i=1}^{N} \exp \left[-s \exp \left(\frac{z_{i}}{\boldsymbol{\xi}}\right)\right]} \\
& \times \exp \left(-\frac{(\boldsymbol{z}-\boldsymbol{\mu})^{T} C_{z}^{-1}(\boldsymbol{z}-\boldsymbol{\mu})}{2}\right) d \boldsymbol{z}
\end{aligned}
$$

where $s$ is the transform variable in the Laplace domain.

To de-correlate (11) as in [9], we make the variable transformation $\mathbf{z}=\sqrt{2} \mathbf{C}_{\mathbf{Z}}{ }^{1 / 2} \mathbf{x}+\boldsymbol{\mu}$ and (11) becomes

$$
\begin{aligned}
M_{\gamma_{N}}(s)= & \int_{-\infty}^{\infty} \frac{1}{\pi^{N / 2}} \prod_{i=1}^{N} \exp \left[-s \exp \left(\frac{\sqrt{2}}{\xi} \sum_{j=1}^{N} c_{i j} x_{j}+\frac{\mu_{i}}{\boldsymbol{\xi}}\right)\right] \\
& \times \exp \left(-\boldsymbol{x}^{T} \boldsymbol{x}\right) d \boldsymbol{x}
\end{aligned}
$$

where $c_{i j}$ is the $(i, j)$ element of $\mathbf{C}_{\mathbf{Z}}^{1 / 2}$, which is obtained from $C_{Z}$ using Cholesky decomposition.

The integral in (12) has the suitable form for GaussHermite expansion approximation [13] for the MGF of the sum of $N$ correlated lognormal SNRs, which is [9]

$$
\begin{aligned}
M_{\gamma_{N}}\left(s, \boldsymbol{\mu}, \boldsymbol{C}_{z}\right) \approx & \sum_{n_{N}=1}^{N_{p}} \ldots \sum_{n_{1}=1}^{N_{p}} \frac{w_{n_{1}} \ldots w_{n_{N}}}{\pi^{N / 2}} \\
& \times \exp \left[-s \sum_{i=1}^{N} \exp \left(\frac{\sqrt{2}}{\xi} \sum_{j=1}^{N} c_{l j} a_{n j}+\frac{\mu_{i}}{\xi}\right)\right],
\end{aligned}
$$


in which $w_{n}$ and $a_{n}$ are, respectively, the weights and the abscissas of the Gauss-Hermite polynomial. The approximation becomes more and more accurate with increasing approximation order $N_{\mathrm{p}}$.

We use the simple decreasing correlation model in [14] for shadow fading. The covariance matrix of the channel SNRs, assuming independent and identically distributed (i.i.d.) channels, is

$$
\sum_{\mathrm{Ln}}(i, j)=\operatorname{Cov}\left(\gamma_{i}, \gamma_{j}\right)=\sigma_{i j}^{* 2}=\sigma^{* 2} \rho^{|i-j|}
$$

in which $\sigma^{2}$ is the variance of per channel SNR and $\rho$ is the correlation coefficient of two adjacent channels.

In the Appendix we show how the Gaussian covariance matrix $\mathbf{C}_{\mathbf{Z}}$ is calculated from the given lognormal covariance matrix $\sum_{\mathrm{Ln}}$ in (14).

\subsubsection{Estimate of sum of lognormal RVs as a single lognormal RV}

In this section, we approximate the sum of $N$-correlated lognormal SNRs by another single lognormal SNR, $\hat{\gamma}_{\ln }=10^{0.1 \hat{X}}$, where $\hat{X}^{\propto} N\left(\hat{\mu}_{X}, \hat{\sigma}_{X}^{2}\right)$. In [9], by matching the MGF of the approximation with the MGF of the lognormal sum $\gamma_{N}$ in (13) at two different positive real values $s_{1}$ and $s_{2}$, a system of two simultaneous equations as in (15) is obtained which can then be used to solve for $\hat{\mu}_{X}$ and $\hat{\sigma}_{X}^{2}$

$$
\begin{aligned}
& \sum_{n=1}^{N_{\mathrm{p}}} w_{n} \exp \left[-s_{i} \exp \left\{\left(a_{n} \hat{\sigma}_{X} \sqrt{2}+\hat{\mu}_{X}\right) / \xi\right\}\right] \\
& =\sqrt{\pi} M_{\gamma_{N}}\left(s_{i}, \boldsymbol{\mu}, \boldsymbol{C}\right), i=1,2 .
\end{aligned}
$$

The weakness in using the two-point MGF-matching method is that it is highly sensitive to the chosen matching points. Furthermore, the method does not guarantee conservation of signal power across the MRC combiner, i.e., equal system average power gain at both sides of the combiner. In this paper, we propose to use this 'lossless' MRC principle to improve the accuracy of the selection of the two matching points. This is a significant contribution of our paper.

We can simplify the problem by assuming a microdiversity environment [15]; i.e., all repetition subchannels experience the same shadowing having $L N\left(\mu_{Z}, \sigma_{Z}^{2}\right)$ distribution, thus have the same local average power. This assumption is quite reasonable for adjacent subchannels within an OFDMA frame. The average SNR of each diversity branch at the input to the MRC receiver is

$$
\bar{\gamma}_{z}=\exp \left[\frac{\mu_{z}}{\xi}+\frac{1}{2}\left(\frac{\sigma_{z}}{\xi}\right)^{2}\right]
$$

The principle of a lossless MRC thus gives the corresponding SNR at the output of the receiver as

$$
\hat{\bar{\gamma}}_{\mathrm{Ln}}=\exp \left[\frac{\hat{\mu}_{X}}{\xi}+\frac{1}{2}\left(\frac{\hat{\sigma}_{X}}{\xi}\right)^{2}\right]=N \bar{\gamma}_{Z} .
$$

Equation 17 provides a valid and reliable equation for iteratively improving the accuracy of the locations of the two MGF matching points. The percentage error of power loss is defined as

$$
\% \text { Error }=100 \frac{N \bar{\gamma}_{Z}-\hat{\bar{\gamma}}_{\text {Ln }}}{N \bar{\gamma}_{Z}} \text {. }
$$

A simple iterative search algorithm for the two matching locations in (15) is carried out until the power loss decreases to a specified error threshold which is set at $0.5 \%$ in this paper. The result of the MGF matching is reported in Table 2. The matching in [9] does not observe the power conservation, and all the matching pairs suggested in the paper result in very large power losses.

Finally, the estimated PDF of the SNR from the diversity combiner is

$$
\hat{f}_{\text {lognormal,MRC }}(\gamma)=\frac{1}{\gamma} \frac{\xi}{\hat{\sigma}_{X} \sqrt{2 \pi}} \exp \left(-\frac{\left(10 \log _{10} \gamma-\hat{\mu}_{X}\right)^{2}}{2 \hat{\sigma}_{X}^{2}}\right) .
$$

\begin{tabular}{|c|c|c|c|c|c|}
\hline Number & $\begin{array}{c}\left(s_{1}, S_{2}\right) ;\left(\hat{\mu}_{X}, \hat{\sigma}_{X}\right) \\
\text { in dB from } \\
\text { two-point } \\
\text { MGF matching }\end{array}$ & $\begin{array}{c}\hat{\hat{Y}}_{L n} \\
\rho=0 \\
(\mathrm{~dB})\end{array}$ & $\begin{array}{c}G_{\mathrm{r}} \\
\rho=0 \\
(\mathrm{~dB})\end{array}$ & $\begin{array}{c}\hat{\bar{Y}}_{L n} \\
\rho=0.2 \\
\text { (dB) }\end{array}$ & $\begin{array}{c}G_{\mathrm{r}} \\
\rho=0.2 \\
(\mathrm{~dB})\end{array}$ \\
\hline \multirow[t]{2}{*}{1} & & 43.31 & 1.00 & 43.31 & 1.00 \\
\hline & & & (0) & & (0) \\
\hline \multirow[t]{2}{*}{2} & $(0.001,2.0)$ & 30.73 & 18.11 & 33.05 & 10.61 \\
\hline & $(2.2528,6.7572)$ & & (12.58) & & (10.26) \\
\hline \multirow[t]{2}{*}{4} & $(0.001,2.7160)$ & 20.54 & 189.23 & 23.44 & 97.05 \\
\hline & $(2.7377,6.7534)$ & & (22.77) & & (19.87) \\
\hline \multirow[t]{2}{*}{6} & $(0.021,1.179)$ & 15.82 & 561.05 & 17.52 & 379.31 \\
\hline & $(2.7377,6.7534)$ & & (27.49) & & (25.79) \\
\hline
\end{tabular}

For the case of no-diversity $(N=1)$ from (4) (for $M=4)$ and (7),

$$
\begin{aligned}
\mathrm{BER}_{\text {lognormal }, \mathrm{QPSK}}= & \int_{0}^{\infty} \mathrm{BER} \text { AWGN,QPSK}(\gamma) \frac{1}{\gamma} \frac{\xi}{\sigma_{z} \sqrt{2 \pi}} \\
& \times \exp \left(-\frac{\left(10 \log _{10} \gamma-\mu_{z}\right)^{2}}{2 \sigma_{z}^{2}}\right) d \gamma .
\end{aligned}
$$

Table 2 Estimated distribution parameters and repetition coding gain

Estimated distribution parameters from MGF matching and required average SNR $\hat{\hat{Y}}_{L n}$ and repetition coding gain for $\mathrm{BER}=10^{-5}$ in correlated lognormal fading channels with $\sigma_{z}=8 \mathrm{~dB}$. 
By a change of variable,

$$
\frac{10 \log _{10} \gamma-\mu_{Z}}{\sigma_{z} \sqrt{2}}=u \Leftrightarrow \gamma=\exp \left(\frac{\mu_{Z}}{\xi}+\frac{\sqrt{2} \sigma_{z}}{\xi} u\right),
$$

(20) can be reduced to

$$
\operatorname{BER}_{\text {lognormal,QPSK }}=\frac{1}{\sqrt{\pi}} \int_{0}^{\infty} \operatorname{BER}_{\mathrm{AWGN}, \mathrm{QPSK}}\left(\gamma_{z}(u)\right) e^{-u^{2}} d u,
$$

where $\gamma_{z}(u)=\exp \left(\frac{\mu_{z}}{\xi}+\frac{u \sigma_{z} \sqrt{2}}{\xi}\right)$ is the argument of BER $_{\text {AWGN,QPSK}}($.$) in (4). The above expression for BER$ can then be accurately approximated by an $N_{\mathrm{p}}$-order Gauss-Hermite polynomial expansion as given in (21)

$$
\mathrm{BER}_{\text {lognormal, }, \mathrm{PPK}}=\frac{1}{\sqrt{\pi}} \sum_{n=1}^{N_{p}} w_{n} \operatorname{BER}_{\mathrm{AWGN}, \mathrm{QPSK}}\left(\gamma z\left(a_{n}\right)\right) .
$$

When we use $N_{\mathrm{p}}=12$, the BER results in (20) and (21) are almost the same.

For the case of $N>1$ from (4) and (19), we obtain

$$
\begin{aligned}
\mathrm{BER}_{\text {lognormal,QPSK,MRC }} & =\int_{0}^{\infty} \operatorname{BER}_{\mathrm{AWGN}, \mathrm{QPSK}}(\gamma) \\
& \times \hat{f}_{\text {lognormal,MRC }}(\gamma) d \gamma,
\end{aligned}
$$

and we obtain (23) below in a similar way in which we obtain (21) above, i.e.,

$$
\begin{aligned}
\mathrm{BER}_{\operatorname{lognormal}, \mathrm{QPSK}, \mathrm{MRC}}= & \frac{1}{\sqrt{\pi}} \sum_{n=1}^{N_{\mathrm{p}}} w_{n} \mathrm{BER}_{\mathrm{AWGN}, \mathrm{QPSK}} \\
& \left(\hat{\gamma}_{X}\left(a_{n}\right)\right)
\end{aligned}
$$

where $\hat{\gamma}_{X}\left(a_{n}\right)=\exp \left(\left(\hat{\mu}_{X}+a_{n} \hat{\sigma}_{X} \sqrt{2}\right) / \xi\right)$.

In Figure 1 we plot BER as a function of the average symbol SNR per subchannel with the signal being subjected to correlated lognormal fading, as calculated from (21) for $N=1$ and from (23) for the case of $N>1$ i.i. d. repetition-coded channels with correlation $\rho=0.2$. It is reasonable that we cannot expect the calculated BER and the Monte Carlo simulated BER to be the same, simply because the calculated BER is only approximated first by using MGF matching technique then by using GaussHermite polynomial approximation.

We define repetition coding gain $\left(G_{\mathrm{r}}\right)$ as the ratio of the average SNR, $\hat{\gamma}_{\mathrm{L} n}$, without repetition coding $(N=1)$ to that with repetition coding $(N>1)$ required for the same given target BER $=10^{-5}$.

In Table 2 we list the required average symbol SNR per channel, $\hat{\bar{\gamma}}_{\mathrm{Ln}}$, to meet the target BER $=10^{-5}$ calculated from
(21) and (23) for QPSK and fixed Gaussian standard deviation $\sigma_{Z}=8 \mathrm{~dB}$ for the lognormal channel. The corresponding repetition coding gain $G_{r}$ for different values of repetition is also listed in Table 2 . The channel correlation with $\rho=0.2$ is seen from Table 2 to have reduced $G_{\mathrm{r}}$ by 2 to $3 \mathrm{~dB}$. This degradation increases at 5 to $6 \mathrm{~dB}$ when we increase the correlation to $\rho=0.6$.

\subsection{Independent composite Rayleigh-lognormal (Suzuki) fading channels}

As has been mentioned in the Section 1, the exact modeling of the fading channels is not the main theme of our paper. There are two justified reasons why in this section we assume that repetition channels are uncorrelated for simplicity. One is the lack of a computationally efficient closed-form expression for BER of correlated composite Rayleigh-lognormal channels using MRC diversity reception and two is, as will be shown in Section 4.2.1 for lognormal channels, that the correlation between repetition diversity channels has little effect on the proposed 10-state FSMC model.

\subsubsection{Physical model for composite Rayleigh-lognormal fading channels}

In [11] a simple physical model for urban mobile radio propagation is presented in which the main wave from the transmitter to the local cluster of buildings in the neighborhood of the receiver traverses a path subject to cascaded reflections and/or diffractions by natural and man-made obstructions. After arrival at the local cluster, the main wave is scattered into multipaths which arrive at the receiver with approximately the same delay and amplitude but with different random phases. Therefore, the signal power gain of the transmitter-tocluster main path is modeled as having lognormal distribution, $p_{\mathrm{Ln}}$, because of the multiplicative effects of reflections and/or diffractions, while that of the local multipaths are modeled as Rayleigh distributed, $p_{R}$, due to additive scattering effects. This model allows us to obtain the marginal probability density distribution for signal-to-noise ratio of a composite Rayleigh-lognormal fading channel, suitable for mobile radio propagation between the base station and a mobile receiver in urban areas, as [15]

$$
\begin{aligned}
f_{\mathrm{R}-\mathrm{Ln}}(\gamma)= & \int_{0}^{\infty} f_{R}(\gamma \mid x) f_{\mathrm{Ln}}(x) d x \\
= & \int_{0}^{\infty} \frac{1}{x} \exp \left(-\frac{\gamma}{x}\right) \frac{\xi}{x \sigma_{z} \sqrt{2 \pi}} \\
& \times \exp \left[-\frac{\left(10 \log _{10} x-\mu_{z}\right)^{2}}{2 \sigma_{Z}^{2}}\right] d x .
\end{aligned}
$$




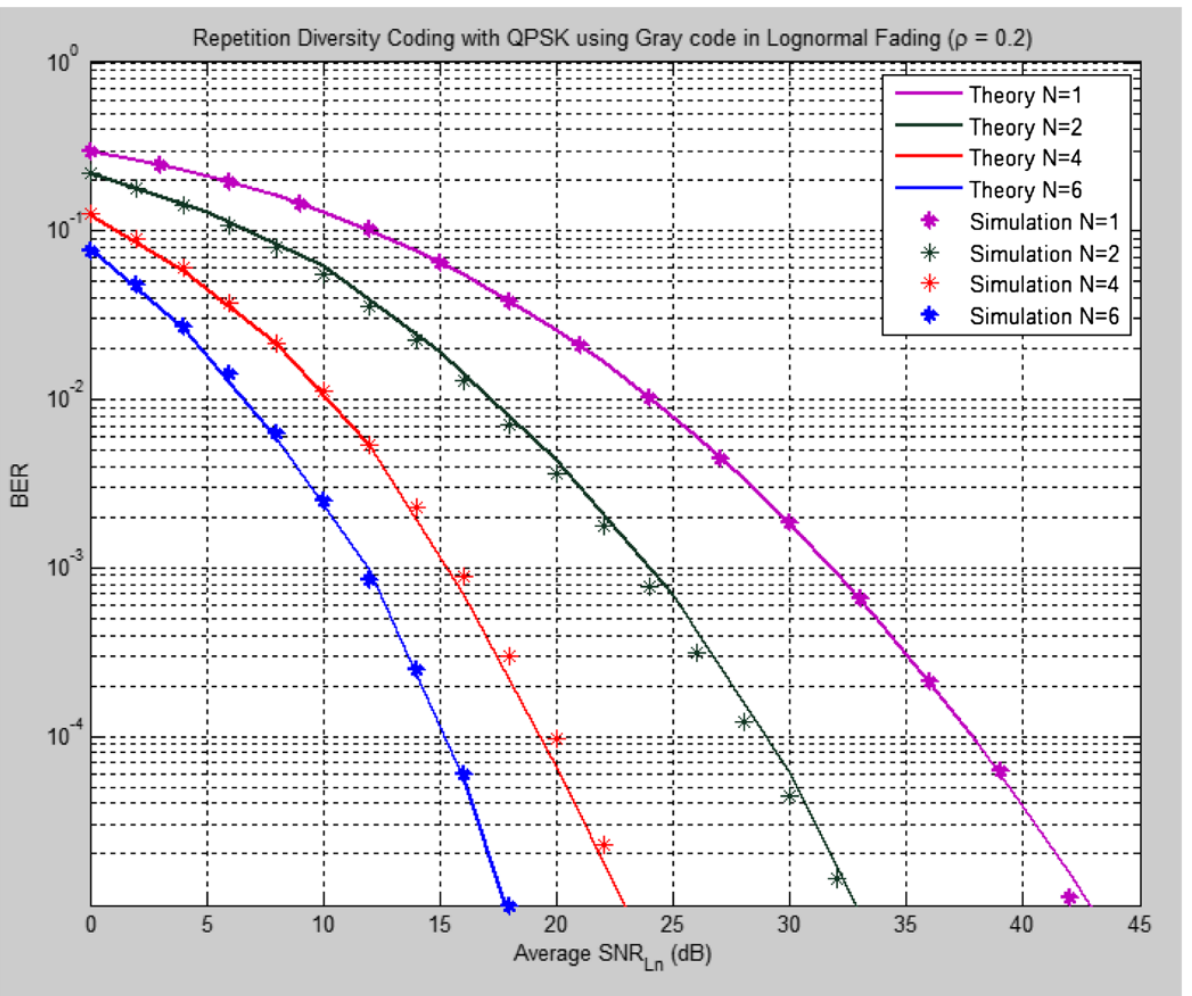

Figure 1 BER versus average SNR per lognormally faded channel $\gamma_{\text {Ln }}$ of QPSK $(\boldsymbol{M}=4)$ using Gray's code. The system uses $N$ th-order repetition coding and maximum ratio combining in correlated lognormal fading channels. Hermite polynomial order $N_{\mathrm{p}}=12 ; \mathrm{Gaussian}$ standard deviation $\sigma_{Z}=8 \mathrm{~dB}$; correlation coefficient $\rho=0.2$.

The distribution in (24) is similar to that given in [11], Equation 3 except that the latter is for Rayleigh distributed signal envelope instead of exponentially distributed signal power in (24).

To develop an expression for the PDF of SNR of a signal using diversity reception in composite Rayleigh-lognormal fading channels and to simulate the scenario using the Monte Carlo technique, it is essential to understand the physical meaning of the fading mechanism. The coherence time of fast Rayleigh fading is a few tens of milliseconds depending on the mobile speed, while the coherence time of slow shadow fading is a few tens of seconds depending on the mobile speed to cover the coherence distance, typically 100 to $200 \mathrm{~m}$ in suburban cells and a few tens of meters in urban cells [14]. Based on the fact of this many-order difference between the two coherence times, the marginal probability density function of the composite Rayleighlognormal channel is derived in $[11,15]$ by equating the local average SNR of the much faster Rayleigh fading signal to the instantaneous SNR of the much slower arriving lognormal signal. This implies first a complete transfer, i.e., a transition, of signal power from the main arriving lognormal signal to the local multipath channel, and second, no significant loss of power in the local multipath channel, i.e., the average power gain of the local Rayleigh fading channel can be assumed as unity. It is therefore interesting to note that the composite distribution in (24) is, in fact, the PDF of the power gain of the product channel $\left|h_{R}-\mathrm{Ln}\right|^{2}=\left|h_{\mathrm{R}}\right|^{2}$ $\left|h_{\mathrm{Ln}}\right|^{2}$ of two cascaded channels $h_{\mathrm{R} i}$ and $h_{\mathrm{Ln} i}$ in Figure 2 . Since $p_{R}\left(\left|h_{R}\right|^{2}\right.$ is exponentially distributed with average $\left.E_{\mathrm{L}}\left|h_{\mathrm{R}}\right|^{2}\right\lrcorner=1$ regardless of the frequency, i.e. frequency non-selective, and $p_{\mathrm{R}}\left(\left|h_{\mathrm{Ln}}\right|^{2}\right)$ is frequency non-selective lognormal distributed as given in (7), the PDF of the product channel, $p_{\mathrm{R}}-\mathrm{Ln}\left(\left|h_{\mathrm{R}}-\mathrm{Ln}\right|^{2}\right)$ as given in (24), is effectively frequency non-selective.

We model the repetition coding as shown in Figure 2 in which the signal path from each subchannel is modeled according to (24). Thus in the general propagation environment, the local Rayleigh-faded signals from different repetition subchannels arrive at the diversity combiner with different local average powers. Unfortunately, while the sum of many lognormal functions is another lognormal function, this is not true for Rayleigh distribution. We can simplify the problem by assuming a microdiversity environment [15], i.e., all repetition subchannels experience the same shadowing having $L N$ $\left(\mu_{Z}, \sigma_{Z}^{2}\right)$ distribution, thus have the same local average power. 


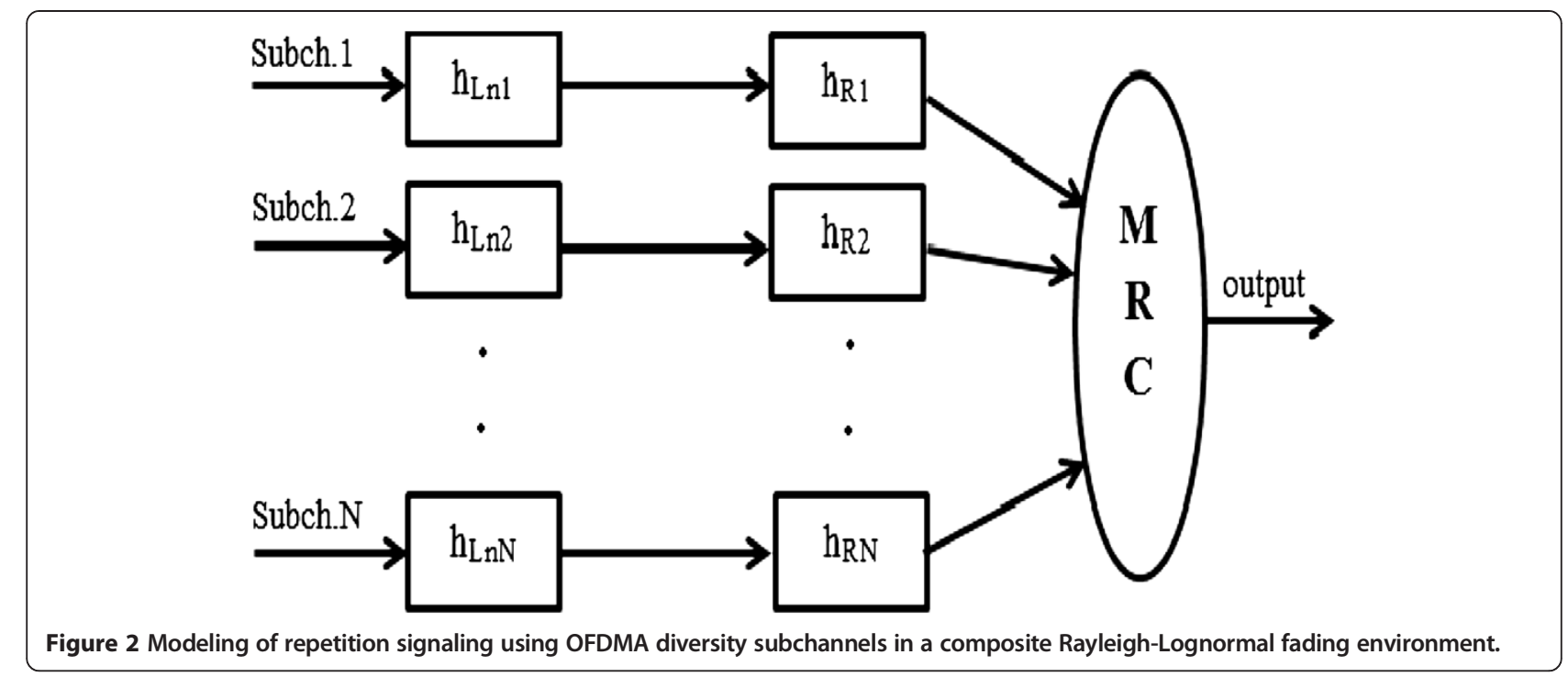

The PDF of the output SNR from the MRC combiner when input is from $N$ i.i.d. diversity subchannels subjected to Rayleigh fading with average SNR $\bar{\gamma}_{\mathrm{R}}$ is given as [12]

$$
\mathrm{BER}_{\text {Rayleigh,QPSK,MRC }}=\frac{1}{2}\left[1-\frac{\mu}{\sqrt{2-\mu^{2}}} \sum_{k=0}^{N-1}\left(\begin{array}{c}
2 k \\
k
\end{array}\right)\left(\frac{1-\mu^{2}}{4-2 \mu^{2}}\right)^{k}\right]
$$

in which $\mu=\sqrt{\frac{\bar{\gamma}_{\mathrm{R}}}{1+\bar{\gamma}_{\mathrm{R}}}}$.

Therefore, in a similar way to the derivation of the PDF in (24) of a product of two random variables, the marginal PDF of the resultant SNR of an $N$-repetition-coded signal subject to composite Rayleigh-lognormal fading can be readily obtained, using Jacobian transformation technique, as

$$
\begin{aligned}
f_{\mathrm{R}-\mathrm{Ln}, \mathrm{MRC}}(\gamma)= & \int_{0}^{\infty} f_{\text {Rayleigh,MRC }}(\gamma \mid x) f_{\text {lognormal }}(x) d x \\
f_{\mathrm{R}-\mathrm{Ln}, \mathrm{MRC}}(\gamma)= & \frac{\xi}{\sigma_{z} \sqrt{2 \pi}} \frac{\gamma^{N-1}}{\Gamma(N)} \int_{0}^{\infty} \frac{1}{x^{N+1}} \exp \left(-\frac{\gamma}{x}\right) \\
& \times \exp \left[-\frac{\left(10 \log _{10^{x}} x-\mu_{z}\right)^{2}}{2 \sigma_{z}^{2}}\right]
\end{aligned}
$$

which takes a form similar to that in [15], Equation 1.

The bit error rate of QPSK signal using repetition diversity coding in a composite Rayleigh-lognormal fading channel is

$$
\operatorname{BER}_{\mathrm{R}-\mathrm{Ln}, \mathrm{QPSK}, \mathrm{MRC}}=\int_{0}^{\infty} \operatorname{BER}_{\mathrm{AWGN}, \mathrm{QPSK}}(\gamma) f_{\mathrm{R}-\mathrm{Ln}, \mathrm{MRC}}(\gamma) d \gamma .
$$

By inserting (26) into (27) and by some rearrangement, we can arrive at

$$
\begin{aligned}
\text { BER }_{\mathrm{R}-\mathrm{Ln}, \mathrm{QPSK}, \mathrm{MRC}}= & \int_{0}^{\infty}\left[\int_{0}^{\infty} \operatorname{BER}_{\mathrm{AWGN}, \mathrm{QPSK}}(\gamma) \frac{\gamma^{N-1}}{\Gamma(N) x^{N}} e^{-\frac{\gamma}{x}} d \gamma\right] \\
& \times f_{\mathrm{R}-\mathrm{Ln}}(x) d x .
\end{aligned}
$$

The term in the square brackets can be identified as BER of QPSK using Gray coding and MRC receiver in Rayleigh fading channel with average SNR $\bar{\gamma}=x$ (see (25)). Moreover, by a change of variable as done for (20) above, (28) can be reduced to the form in (29) below

$$
\begin{aligned}
\mathrm{BER}_{\mathrm{R}-\mathrm{Ln}, \mathrm{QPSK}, \mathrm{MRC}}= & \frac{1}{\sqrt{\pi}} \int_{0}^{\infty} \mathrm{BER}_{\text {Rayleigh, } \mathrm{QPSK}, \mathrm{MRC}} \\
& \left(\bar{\gamma}_{R}(z)\right) e^{-Z^{2}} d z,
\end{aligned}
$$

where $\bar{\gamma}_{\mathrm{R}}(z)=\exp \left(\mu_{z} / \xi+z \sigma_{z} \sqrt{2} / \xi\right)$ is the argument of

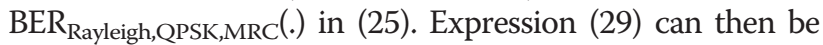
accurately approximated by an $N_{\mathrm{p}}$-order Gauss-Hermite polynomial expansion as in (30) below:

$$
\begin{aligned}
\mathrm{BER}_{\mathrm{R}-\mathrm{Ln}, \mathrm{QPSK}, \mathrm{MRC}}= & \frac{1}{\sqrt{\pi}} \sum_{n=1}^{N_{\mathrm{p}}} w_{n} \mathrm{BER}_{\text {Rayleigh, } \mathrm{QPSK}, \mathrm{MRC}} \\
& \left(\bar{\gamma}_{\mathrm{R}}\left(a_{n}\right)\right)
\end{aligned}
$$

when $N_{\mathrm{p}}=12$, and (30) and (27) both give almost exactly the same BER after the latter is adjusted for Gray coding. Thus (30), by avoiding the double integration in (28), provides a much faster way to calculate 


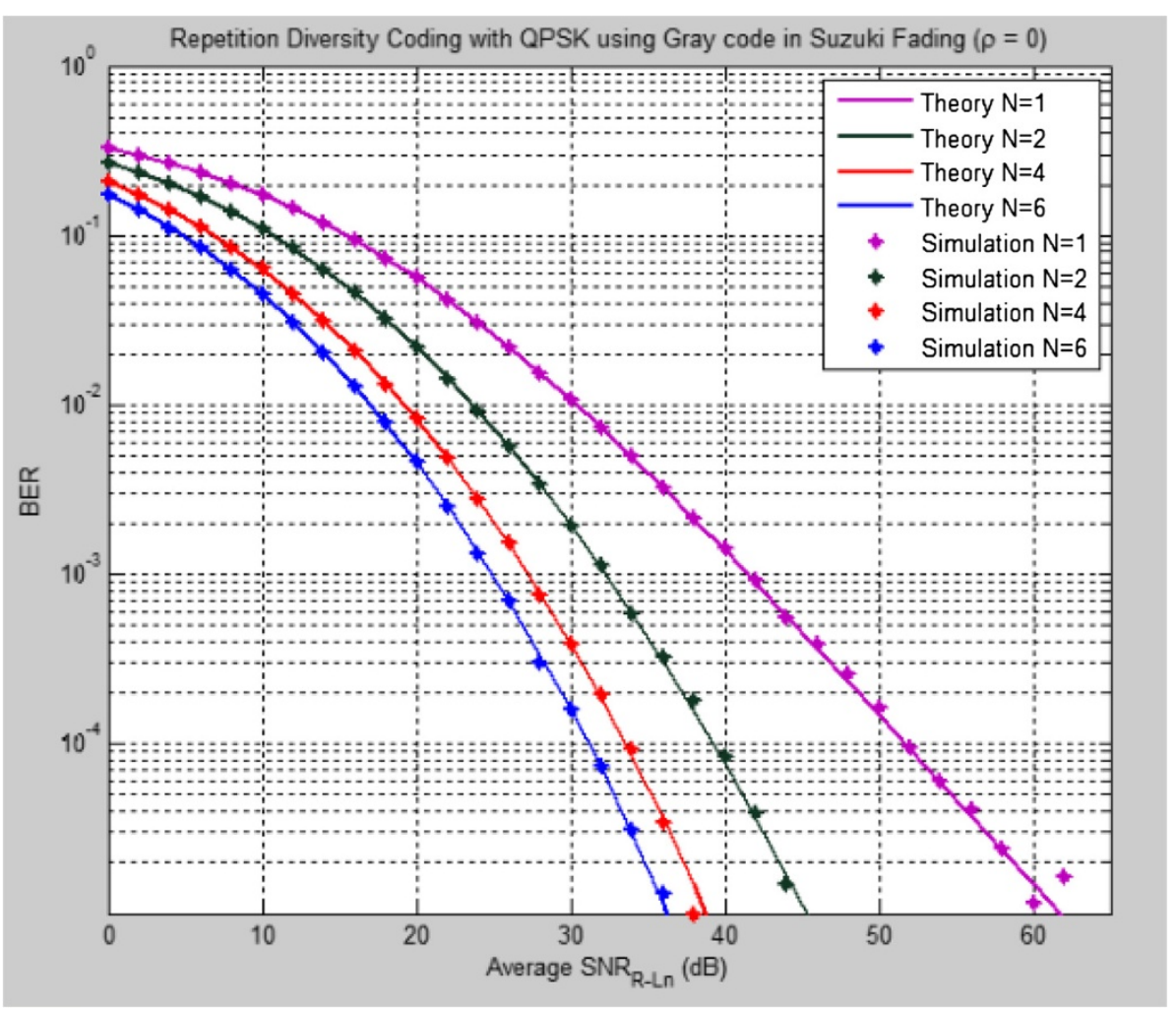

Figure 3 BER versus average SNR $\bar{\gamma}_{R-L n}$ per composite Rayleigh-lognormally faded channel of QPSK.

BER of QPSK signal using MRC diversity reception in Suzuki fading channels. In Figure 3 we plot the BER as a function of the average symbol SNR, $\bar{\gamma}_{\mathrm{R}-\mathrm{Ln}}$, of each subchannel signal being subjected to composite Rayleigh-lognormal fading. The system uses $N$ th-order repetition coding and maximum ratio combining, Hermite polynomial order $N_{\mathrm{p}}=12$, Gaussian standard deviation $\sigma_{Z}=8 \mathrm{~dB}$.

Again, we define repetition coding gain, $G_{r}$, as the ratio of the required average SNR to meet a given BER target of $10^{-5}$ when $\mathrm{RC}$ is not used to that when $\mathrm{RC}$ is used. The required average SNR calculated from (30) and the corresponding RC gain for the different number of repetitions are listed in Table 3.

\section{The 10-state model for the AMC scheme with repetition diversity coding}

4.1 State partition for the AMC scheme in mobile WiMAX

As mentioned in Section 1, the AMC scheme forms a discrete set of combined modulation and coding $s_{j}=\left\{M_{j}\right.$, $\left.R_{j}, x_{j}\right\}$ specified by the corresponding standard. By partitioning the range of the received SNR into a finite number of intervals to match the discrete set of modulation and coding, a finite-state Markov channel (FSMC) model can be constructed for the implementation of the AMC scheme in fading wireless channels. In this section we use mobile WiMAX as a case study, but the approach can be generalized to design power control algorithm for other wireless communication systems using AMC under fading conditions.

In adaptive modulation and coding, at each symbol time, the wireless system assigns a state $s_{j}=\left\{M_{j}, R_{j}, x_{j}\right\}$ and the associated transmit power to a received SNR $\gamma$. Therefore, as SNR varies with the fading condition, BER will change accordingly. The aim of power control is to adapt the transmit power to the instantaneous received SNR so that BER stays at the given target level in all states. The 10-state combined modulation and coding rates in mobile WiMAX are calculated as follows [6]:

$$
M_{j}=\left\{\begin{array}{cl}
4 & j=1,2,3,4,5 \\
16 & j=6,7 \\
64 & j=8,9,10
\end{array}\right.
$$

Table 3 Required average SNR $\bar{\gamma}_{\mathrm{R} \text {-Ln }}$ and repetition coding gain for BER $=10^{-5}$

\begin{tabular}{lcc}
\hline Number & $\overline{\boldsymbol{V}}_{\mathrm{R}-\mathrm{Ln}}(\mathrm{dB})$ & $\boldsymbol{G}_{\mathbf{r}}$ linear ratio unit and $(\mathrm{dB}), \boldsymbol{\rho}=\mathbf{0}$ \\
\hline 1 & 61.32 & $1.00(0)$ \\
2 & 45.10 & $41.88(16.22)$ \\
4 & 38.66 & $184.50(22.66)$ \\
6 & 36.06 & $335.74(25.26)$ \\
\hline
\end{tabular}


and

$$
M_{R_{j}}=\left(M_{j}\right)^{R_{j}}
$$

with the effective coding rate $R_{j}=\mathrm{RS}-\mathrm{CC}$ coding rate divided by the number of repetitions, i.e.,

$$
R_{j}=[1 / 12,1 / 8,1 / 4,1 / 2,3 / 4,1 / 2,3 / 4,2 / 3,3 / 4,5 / 6]
$$

and

$$
\begin{gathered}
M_{R j}=[1.1225,1.1892,1.4142,2,2.8284, \\
4,8,16,22.6274,32] .
\end{gathered}
$$

Thus, for each value of the instantaneous SNR, $\gamma$, the AMC algorithm will decide which $M$-QAM, what coding rate, what repetition rate, and what associated transmit power to use.

\subsection{Optimal power adaptation in M-QAM}

In this section a brief review and explanation of the transmit power adaptation technique for $M$-QAM modulation in fading channels $[4,5]$ is presented for continuity and clarity. We want to adapt the transmit power $S(\gamma)$ to the instantaneous value of SNR subject to the average power constraint. The BER upper bound in (5) becomes

$$
\operatorname{BER}_{\mathrm{AWGN}, M-\mathrm{QAM}}(\gamma) \leq K_{\mathrm{B}}(M) \exp \left(-\frac{1.5 \gamma}{M-1} \frac{S(\gamma)}{\bar{S}}\right) .
$$

It can be seen from the bound in (35), for a given value of SNR, $\gamma$, we can adapt both $M(\gamma)$ and $S(\gamma)$ to maintain a given target BER and an average power constraint $\bar{S}$.

The classical approach for constraint optimization of transmit power which maximizes the average spectral efficiency, subject to average power constraint, is to use the Lagrange multiplier technique with a multiplier which can be calculated from the power constraint requirement. This results in the well-known optimal 'water-filling' power adaptation policy in broadband data transmission. Using a similar approach for the problem of optimal power adaption in M-QAM, it has been shown in [4], Equation 25 that the resulting optimal continuous modulation rate for a given value of $\gamma$ is

$$
M(\gamma)=\frac{\gamma}{\gamma_{\beta}}
$$

in which $\gamma_{\beta}$ is the optimized cutoff fade depth that depends on the fading distribution $f(\gamma)$. In the same way as for the Lagrange multiplier $\gamma_{\beta}$ can be calculated from the average power constraint requirement.

In this paper, although the state boundaries and associated modulation and coding rates are fixed, within the state region $j$ the transmit power $S_{j}(\gamma)$ is a continuous function of the SNR. The upper bound for the continuous constellation size in state $j$ for a given target BER can be extracted from (35) as

$$
M_{j}(\gamma) \leq 1+\beta_{j} \frac{S_{j}(\gamma)}{\bar{S}} \gamma \text { for } j=1,2, \ldots 10
$$

in which, by taking both the convolutional coding gain $G_{\mathrm{c}}$ and the repetition coding gain $G_{\mathrm{r}}$ into account, we have

$$
\beta_{j}=-\frac{1.5 G_{\mathrm{c} j} G_{\mathrm{r} j}}{\ln \left(\frac{1}{K_{\mathrm{B}}\left(M_{j}\right)} \mathrm{BER}_{\mathrm{AWGN}}\right)} \text { for } j=1,2,3
$$

and

$$
\beta_{j}=-\frac{1.5 G_{\mathrm{c} j}}{\ln \left(\frac{1}{K_{\mathrm{B}}\left(M_{j}\right)} \mathrm{BER}_{\mathrm{AWGN}}\right)} \text { for } j=4,5, \ldots, 10 .
$$

Once the optimized cutoff phase depth $\gamma_{\beta}$ has been calculated for a given fading distribution $f(\gamma)$, we are ready to quantize the optimal continuous modulation rate in (36) into ten states as specified in Section 4.1 above,

$$
M(\gamma)=M_{\mathrm{R}_{j}} \text { if } M_{\mathrm{R}_{j}} \leq M(\gamma)=\frac{\gamma}{\gamma_{\beta}} \leq M_{\mathrm{R}_{j+1}} .
$$

Accordingly, the range of the SNR is also partitioned into ten regions.

Based on the tight approximation for BER in (35) or equivalent upper bound for modulation rate in (37), a power adaptation policy which maintains a fixed target BER and satisfies the average power constraint, $E[S(\gamma)] \leq \bar{S}$, is proposed in [4] and [5] as

$$
\frac{S_{j}(\gamma)}{\bar{S}}=\left\{\begin{array}{cc}
\left(M_{j}-1\right) \frac{1}{\beta_{j} \gamma}, & M_{\mathrm{R}_{j}} \leq \frac{\gamma}{\gamma_{\beta}} \leq M_{\mathrm{R}_{j+1}} \\
0,(\text { no power }) & 0 \leq \frac{\gamma}{\gamma_{\beta}} \leq M_{\mathrm{R}_{1}}
\end{array}\right.
$$

where $M_{j}$ and $M_{\mathrm{R} j}, j=1,2, \ldots .10$, are given in (33) and (34) respectively, and when $\gamma<\gamma_{\beta} M_{\mathrm{R} 1}$ no power is allocated. The effect of both channel coding and repetition diversity coding has been taken into account by incorporating their respective coding gains into $\beta_{j}$ in (38a) and (38b) which results in a decrease in the adaptive power $S_{j}(\gamma)$ in $(40)$.

The maximized spectral efficiency of the adaptive system for a given fading condition with distribution $f(\gamma)$ is the average of the maximized spectral efficiencies of the $N$ states

$$
E\left[\log _{2} M(\gamma)\right]=\sum_{j=1}^{N} \log _{2}\left(M_{\mathrm{R}_{j}}\right) \operatorname{Pr}\left(M_{\mathrm{R}_{j}} \leq \frac{\gamma}{\gamma_{\beta}} \leq M_{\mathrm{R}_{j+1}}\right)
$$


where $\gamma_{\beta}$ can be calculated from the power constraint requirement

$$
\sum_{j=1}^{N} \int_{M_{j} \gamma_{\beta}}^{M_{j+1} \gamma_{\beta}} \frac{S_{j}(\gamma)}{\bar{S}} f(\gamma) d \gamma=\sum_{j=1}^{N} \frac{M_{j}-1}{\beta_{j}} \int_{M_{j} \gamma_{\beta}}^{M_{j+1} \gamma_{\beta}} \frac{1}{\gamma} f(\gamma) d \gamma=1
$$

\subsubsection{Lognormal fading only}

This is the scenario of a line-of-sight wireless propagation between the transmitter and a receiver in which the radio wave experiences the multiplicative effect of a large number of cascaded obstructions in its path. With the resulting shadowing having the lognormal PDF in (7), the power constraint in (42) becomes

$$
\sum_{j=1}^{N} \frac{M_{j}-1}{\beta_{j}} \int_{M_{j} \gamma_{\beta}}^{M_{j+1} \gamma_{\beta}} \frac{1}{\gamma^{2}} \frac{\zeta}{\sigma_{z} \sqrt{2 \pi}} \exp \left\{-\frac{\left(10 \log _{10} \gamma-\mu_{z}\right)^{2}}{2 \sigma_{z}^{2}}\right\} d \gamma=1
$$

in which $\mu_{Z}$ and $\sigma_{Z}$ are in decibels.

By letting

$$
x=\frac{\zeta \ln \gamma-\mu_{z}}{\sigma_{z} \sqrt{2}} \Rightarrow x_{j}=\frac{\zeta \ln \left(M_{j} \gamma_{\beta}\right)-\mu_{z}}{\sigma_{z} \sqrt{2}}
$$

we can reduce (43), by using [16], 3.322.1, p. 336, to

$$
\begin{gathered}
\frac{1}{\sqrt{\pi}} \exp \left(-\frac{\mu_{z}}{\xi}\right) \sum_{j=1}^{N} \frac{M_{j}-1}{\beta_{j}} \int_{x_{j}^{2}}^{x_{j+1}} \exp \left\{-\left(x^{2}+\frac{\sigma_{z} \sqrt{2}}{\xi} x\right)\right\} \\
\times d x=\frac{1}{2} \exp \left\{-\frac{\mu_{z}}{\zeta}+\frac{\sigma_{z}^{2}}{2 \zeta^{2}}\right\} \sum_{j=1}^{N} \frac{M_{j}-1}{\beta_{j}} \\
\times\left\{\Phi\left(\frac{\sigma_{z}}{\zeta \sqrt{2}}+x_{j+1}\right)-\Phi\left(\frac{\sigma_{z}}{\zeta \sqrt{2}}+x_{j}\right)\right\}=1
\end{gathered}
$$

in which $\Phi()=.\operatorname{erf}($.$) is the error function.$
Thus, we can numerically solve (45) for the optimized SNR scaling parameter as a function of the lognormal fading depth $\left(\mu_{Z}, \sigma_{Z}^{2}\right)$. In numerically solving (45), it should be noticed from (44) that $\gamma_{\beta} \geq \frac{1}{64}$ $\exp \left(\mu_{z} / \xi\right)$ and the iteration should start with this minimum value for $\gamma_{\beta}$.

For an average SNR of $\bar{\gamma}_{\mathrm{Ln}}=15 \mathrm{~dB}$ and $\sigma_{\mathrm{z}}=8 \mathrm{~dB}$, (42) gives $\gamma_{\beta}=0.4403$ which is practically the same as when there is no correlation between repetition diversity channels. A detailed examination of (45) reveals that the contribution to the right-hand side from the difference of the two $\Phi($.$) functions is non-zero only for j=5$ and $j=7$, i.e., at the transitions where modulation depth changes (see (31)). But at these states, repetition coding does not apply; hence, diversity channel correlation is irrelevant. Using this optimization parameter for the quantization in (39) provides the boundaries of the SNR partition for our 10-state FSMC model as given in Table 4 and illustrated in Figure 4 for the lognormal fading only condition.

\subsubsection{Composite Rayleigh-lognormal (Suzuki) fading}

This is the most realistic scenario and is typical of a link between a base station and a mobile subscriber in a built-up urban area. With the composite Rayleighlognormal PDF in (24), the power constraint in (42)

\begin{tabular}{|c|c|c|c|c|c|c|}
\hline Modulation & State $s_{j}$ & $\mathrm{CC}$ gain $G_{c j}(\mathrm{~dB})$ & $\begin{array}{l}\text { RC gain } G_{r j}(\mathrm{~dB}) \text { in lognormal } \\
\text { fading } \rho=0.2\end{array}$ & $\begin{array}{l}\text { Ten states partition } \\
\text { lognormal fading }\end{array}$ & $\begin{array}{l}\text { RC gain } G_{r j}(\mathrm{~dB}) \text { in } \\
\text { Suzuki fading } \rho=0\end{array}$ & $\begin{array}{l}\text { Ten states partition } \\
\text { Suzuki fading }\end{array}$ \\
\hline \multirow[t]{5}{*}{ QPSK } & 1 & 6.99 & 25.79 & -3.06 & 25.26 & -3.80 \\
\hline & 2 & 6.99 & 19.87 & -2.81 & 22.66 & -3.55 \\
\hline & 3 & 6.99 & 10.26 & -2.05 & 16.22 & -2.80 \\
\hline & 4 & 6.99 & 0 & -0.55 & 0 & -1.29 \\
\hline & 5 & 5.74 & 0 & 0.95 & 0 & 0.21 \\
\hline \multirow[t]{2}{*}{ 16-QAM } & 6 & 6.99 & - & 2.46 & & 1.72 \\
\hline & 7 & 5.74 & - & 5.47 & & 4.73 \\
\hline \multirow[t]{4}{*}{ 64-QAM } & - & 6.99 & - & - & - & - \\
\hline & 8 & 6.02 & - & 8.48 & - & 7.74 \\
\hline & 9 & 5.74 & - & 9.98 & - & 9.24 \\
\hline & 10 & 5.23 & - & 11.49 & - & 10.75 \\
\hline
\end{tabular}
becomes

$$
\begin{aligned}
\sum_{j=1}^{N} \frac{M_{j}-1}{\beta_{j}} \int_{M_{j \gamma_{\beta}}}^{M_{j+1} \gamma_{\beta}} \frac{1}{\gamma} \int_{0}^{\infty} \frac{1}{x} \exp (-\gamma / x) \\
\times \frac{\zeta}{x \sigma_{z} \sqrt{2 \pi}} \exp \left\{-\frac{\left(10 \log _{10} x-\mu_{z}\right)^{2}}{2 \sigma_{z}^{2}}\right\} d x d \gamma=1,
\end{aligned}
$$

in which $\mu_{Z}$ and $\sigma_{Z}$ are in decibels.

Table 4 SNR partition in the 10-state FSMC model for mobile WiMAX in various fading channels 


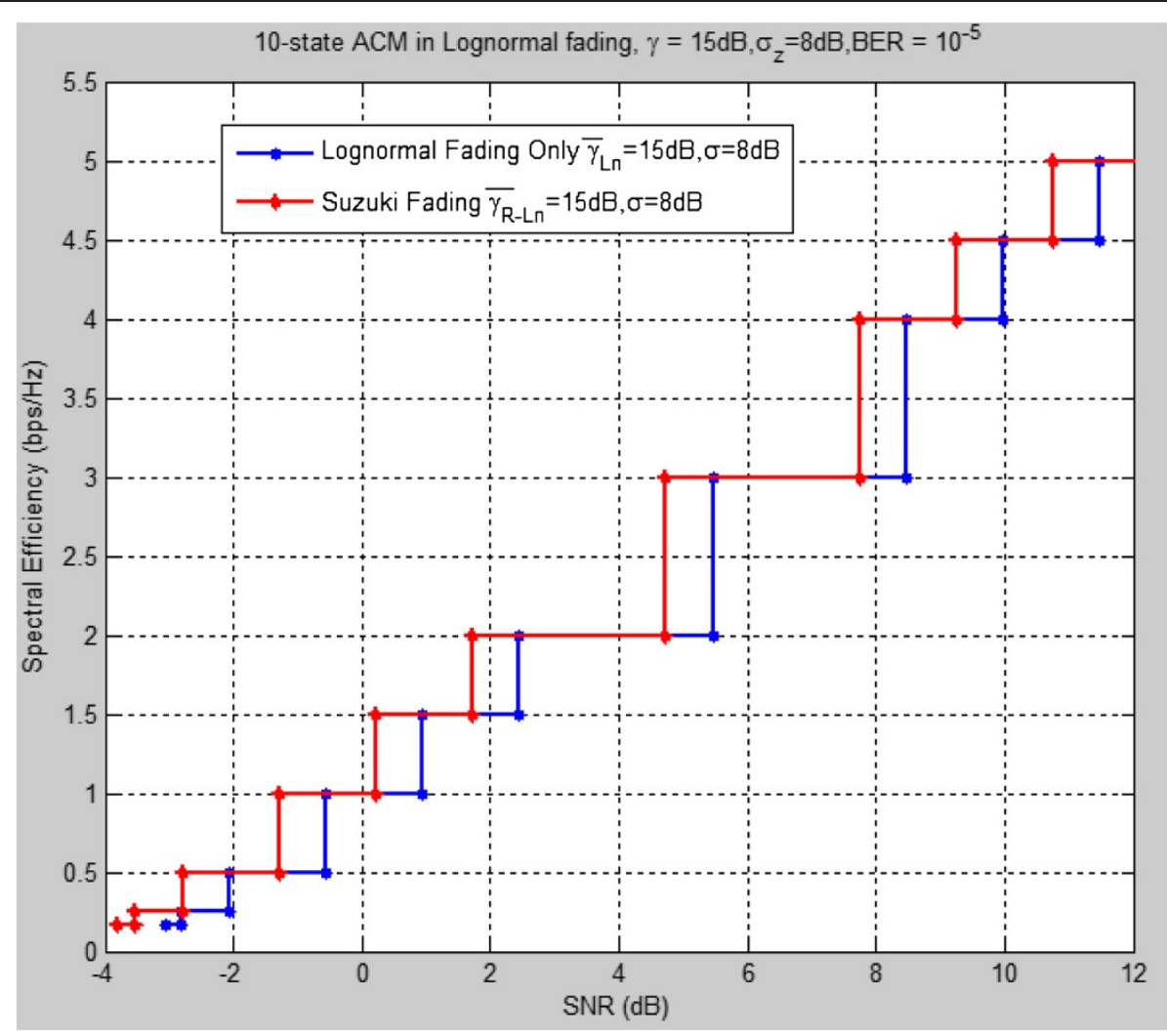

Figure 4 A 10-state SNR partition for the AMC scheme with RDC in mobile WiMAX.

The double integration in (46) can be rearranged as below:

$$
\begin{aligned}
& \sum_{j=1}^{N} \frac{M_{j}-1}{\beta_{j}} \int_{0}^{\infty} \frac{\zeta}{x^{2} \sigma_{z} \sqrt{2 \pi}} \exp \left\{-\frac{\left(10 \log _{10} x-\mu_{z}\right)^{2}}{2 \sigma_{z}^{2}}\right\} \\
& \quad \times\left\{\int_{M_{j} \gamma_{\beta}}^{M_{j+1} \gamma_{\beta}} \frac{1}{\gamma} \exp (-\gamma / x) d \gamma\right\} d x=1 .
\end{aligned}
$$

Then the inner integration $\left\{\int(). d \gamma\right\}$ can be expressed in terms of exponential integral functions [14, 3.354.3], p. 341 and (47) can be reduced to

$$
\begin{gathered}
\sum_{j=1}^{N} \frac{M_{j}-1}{\beta_{j}} \int_{0}^{\infty} \frac{\zeta}{x^{2} \sigma_{z} \sqrt{2 \pi}} \exp \left\{-\frac{\left(10 \log _{10} x-\mu_{z}\right)^{2}}{2 \sigma_{z}^{2}}\right\} \\
\times\left\{E_{1}\left(\frac{M_{j} \gamma_{\beta}}{x}\right)-E_{1}\left(\frac{M_{j+1} \gamma_{\beta}}{x}\right)\right\} d x=1 .
\end{gathered}
$$

Moreover, by a change of variable as in (44), (48) can be further reduced to

$$
\begin{aligned}
& \frac{1}{\sqrt{\pi}} \sum_{j=1}^{N} \frac{M_{j}-1}{\beta_{j}} \int_{-\infty}^{\infty} g(z) \\
& \quad \times\left\{E_{1}\left(M_{j} \gamma_{\beta} g(z)\right)-E_{1}\left(M_{j+1} \gamma_{\beta} g(z)\right)\right\} e^{-z^{2}} d z=1
\end{aligned}
$$

where $g(z)=\exp \left\{-\left(\mu_{z}+z \sigma_{z} \sqrt{2}\right) / \xi\right\}$.

Finally, (49) can be accurately approximated by an $N_{\mathrm{p}^{-}}$ order Gauss-Hermite polynomial as

$$
\begin{aligned}
\frac{1}{\sqrt{\pi}} \sum_{j=1}^{N} \frac{M_{j}-1}{\beta_{j}}\left[\sum _ { n = 1 } ^ { N _ { \mathrm { p } } } w _ { n } g ( a _ { n } ) \left\{E_{1}\left(M_{j} \gamma_{\beta} g\left(a_{n}\right)\right)\right.\right. \\
\left.\left.-E_{1}\left(M_{j+1} \gamma_{\beta} g\left(a_{n}\right)\right)\right\}\right]=1 .
\end{aligned}
$$

Similar to solving (46), we can numerically solve (50) for the optimized SNR scaling parameter $\gamma_{\beta}$ for the composite Rayleigh-lognormal fading case as a function of the lognormal fading depth $\left(\mu_{z}, \sigma_{z}^{2}\right)$. For an average SNR 
$\bar{\gamma}_{\mathrm{R}-\mathrm{Ln}}=15 \mathrm{~dB},(49)$ gives $\gamma_{\beta}=0.3728$ while (50) gives an approximation of $\gamma_{\beta}=0.3714$. Its state boundary is about $1.0 \mathrm{~dB}$ to the left of, i.e., better than, the lognormal fading channel having the same $15 \mathrm{~dB}$ average SNR, as shown in red in Figure 4.

\subsection{Average spectral efficiency in different fading channels}

The spectral performance of the ten states in Figure 4 shows the instantaneous spectral efficiency as a function of the instantaneous signal-to-noise ratio. Since the power adaption algorithm in (40) allocates zero power to state 0 where SNR $\gamma$ falls below $M_{1} \gamma_{\beta}$, it would be interesting to see how much this zero-power state affects the overall average spectral efficiency of the wireless system.

Figure 5 shows the average spectral efficiency performance as a function of the average SNR when the system uses the proposed power adaption scheme in (40). Readers may immediately notice that while in Figure 4 the instantaneous performance of the Suzuki fading channel is about $1.0 \mathrm{~dB}$ better than the lognormal fading channel, its average performance in Figure 5 is the other way round, about $2 \mathrm{~dB}$ worse than that of the latter. The explanation may be found by comparing the probabilities that the SNR of the two channels falls into various states as shown in Figure 6. At low average SNRs, e.g., $2 \mathrm{~dB}$, and more than $50 \%$ of the time, the received Suzuki signal falls into the zero-power state; this figure is just above $45 \%$ for the received lognormal signal. At high average SNRs, there is still a significant probability that the Suzuki signal falls into the zero-power state 0 , e.g., 25\% at average SNR of $12 \mathrm{~dB}$. The probability of the received lognormal faded signal is much lower than that of the Suzuki counterpart in state 0 .

\section{Conclusions}

We have defined and successfully developed expressions for the coding gain of repetition diversity coding and the related 10-state FSMC model for variable power control for AMC used in modern wireless mobile networks operating under the two fading mechanisms: lognormal and composite Rayleigh-lognormal. It is found that the correlation between diversity fading channels, while significantly degrading the BER performance, practically does not affect the proposed power control algorithm and the resulting 10-state FSMC model. By using the power conservation principle across the MRC combiner, an innovative technique is proposed for accurate matching of two MGFs which allows an accurate estimate of the PDF of the SNR at the combiner output. Next, by using the Gauss-Hermite quadrature approximation for integration, we have derived the most computationally fast expressions to date, to the best of our knowledge, for the calculation of BER of QPSK using MRC diversity reception in correlated fading channels and for the efficient computation of the 10-state FSMC

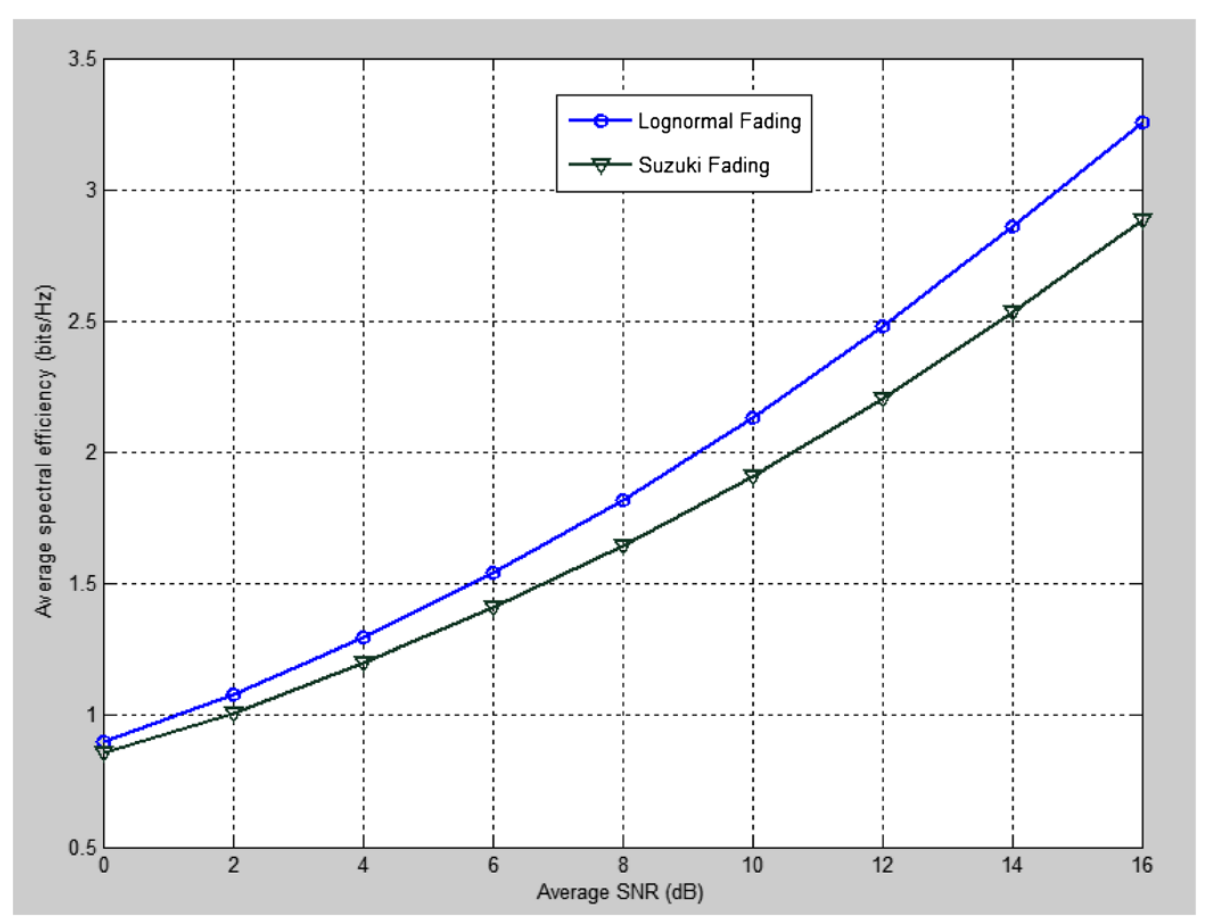

Figure 5 Average spectral efficiency as function of average SNR under lognormal and composite Rayleigh-lognormal fading mechanisms. 


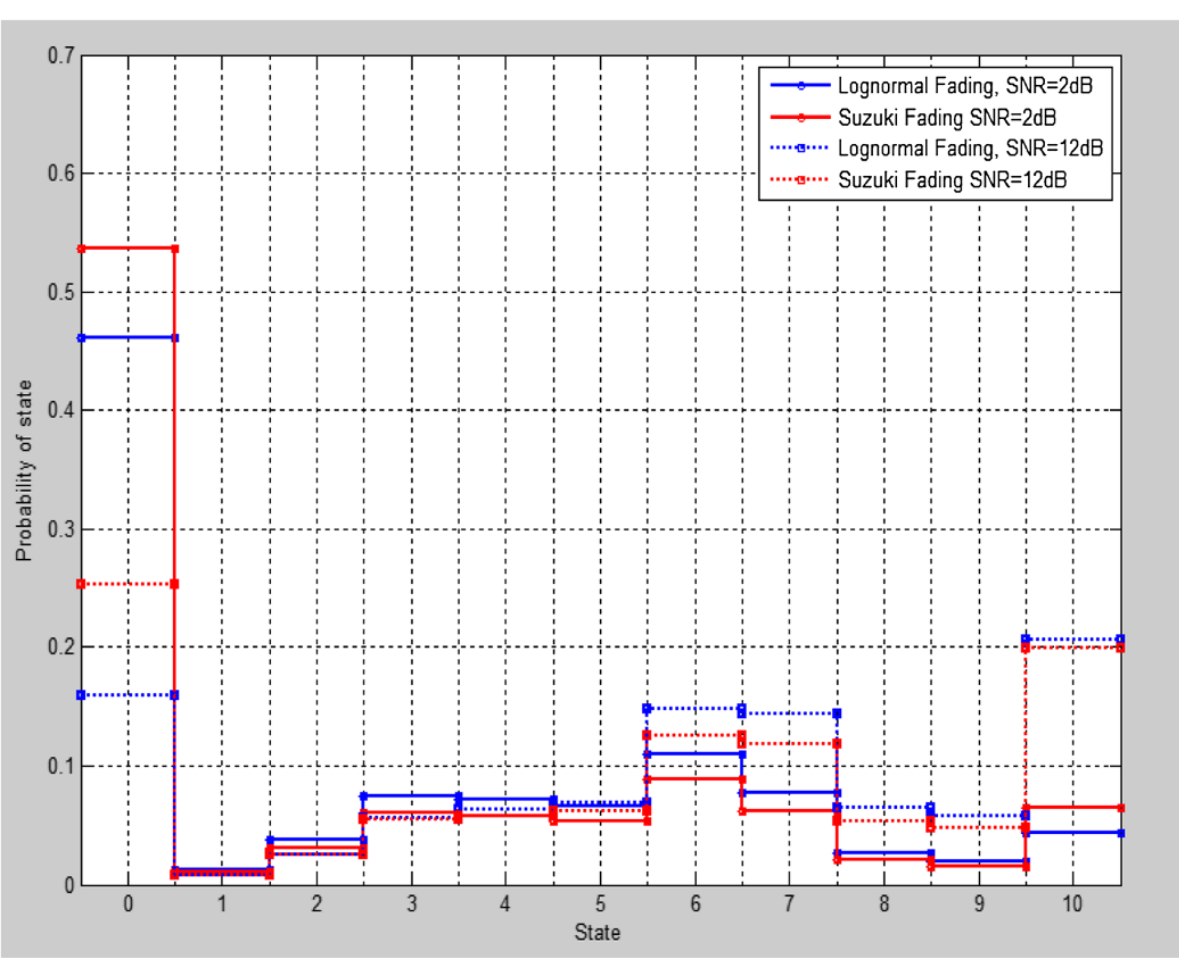

Figure 6 Probability of state visited by channel SNR under lognormal and composite Rayleigh-lognormal fading mechanisms.

model for AMC in mobile WiMAX. The inclusion of repetition coding gain in the transmit power adaptation algorithm for different fading mechanisms and different fading depths has not been done before. From Figure 4, we can observe that the use of RC for QPSK modulation level alone extends the system operation range to almost $4 \mathrm{~dB}$ into very poor fading conditions. In addition, it is interesting to note that based on the same overall average SNR, the composite Suzuki fading model requires approximately $1.0 \mathrm{~dB}$ less power than the lognormal fading model to achieve the same instantaneous spectral efficiency. However, from Figure 5, because of the high probability of the received Suzuki-faded signal falling into the zero-power state, its average spectral efficiency becomes lower than that of the lognormal faded signal.

\section{Appendix}

\section{Calculating Gaussian matrix $C_{z}$ from given lognormal} covariance matrix $\sum$ Ln

We use the simple decreasing correlation model in [14] for shadow fading. The covariance matrix of the channel SNRs, assuming i.i.d. channels, is

$$
\sum_{\mathrm{Ln}}(i, j)=\operatorname{Cov}\left(\gamma_{i}, \gamma_{j}\right)=\sigma_{i j}^{* 2}=\sigma^{* 2} \rho^{|i-j|},
$$

in which $\sigma^{2}$ is the variance of per-channel SNR.
It can be shown that the relationship between the Gaussian channel mean $\mu_{Z}$, variance $\sigma_{Z}^{2}$, covariance $C_{Z}(i, j)$, and the lognormal channel mean $\mu^{*}$, variance $\sigma^{2}$, and covariance $\sum_{\mathrm{Ln}}(i, j)$ can be summarized as below:

$$
\begin{aligned}
\mu^{*} & =E(\gamma)=\bar{\gamma}=e^{\mu_{z}+\sigma_{z}^{2} / 2} \\
\sigma^{* 2} & =\operatorname{Var}(\gamma)=e^{2 \mu_{z}+\sigma_{z}^{2} / 2}\left(e^{\sigma_{z}^{2}}-1\right) \\
& =[E(\gamma)]^{2}\left(e^{\sigma_{z}^{2}}-1\right) .
\end{aligned}
$$

Hence,

$$
\begin{aligned}
& \mu_{z}=E\left(Z_{i}\right)=\ln \left(\frac{\mu^{* 2}}{\sqrt{\mu^{* 2}+\sigma^{* 2}}}\right) \\
& \sigma_{z}^{2}=\operatorname{Var}\left(Z_{i}\right)=\ln \left(1+\frac{\sigma^{* 2}}{\mu^{* 2}}\right) \\
& C_{z}(i, j)=\operatorname{Cov}\left(Z_{i}, Z_{j}\right)=\ln \left(1+\frac{\sigma^{* 2} \rho^{|i-j|}}{\mu^{* 2}}\right) .
\end{aligned}
$$

In this paper, we normalize the channel's mean power gain $\mu^{*}=1$ to avoid dependency on propagation distance and adopt a fixed Gaussian standard deviation $\sigma_{z}=8 \mathrm{~dB}$. 


\section{Abbreviations}

AMC: Adaptive modulation and coding; AWGN: Adaptive white Gaussian noise; BER: Bit error rate; BS: Base station; BTC: Block turbo code; $E_{\mathrm{b}} / N_{0}$ : Bit energy-to-noise ratio; FEC: Forward error correction; FSMC: Finite-state Markov channel; $G_{c}$ and $G_{r}$ : (Error) Coding gain and repetition coding gain; MGF: Moment generating function; MRC: Maximal ratio combining; OFDM: Orthogonal frequency division multiplex; OFDMA: Orthogonal frequency division multiple access; PDF: Probability density function; QAM: Quadrature amplitude modulation; QPSK: Quadrature phase-shift keying; RC: Repetition coding; RS-CC: Reed-Solomon and convolutional code; RV: Random variable; SNR: Signal-to-noise ratio; WiMAX: Worldwide interoperability for microwave access.

\section{Competing interests}

The authors declare that they have no competing interests.

\section{Acknowledgements}

This work was supported by research grants from QG.2014 Projects of the University of Engineering and Technology, Vietnam National University Hanoi. The authors would like to thank the anonymous reviewers for their careful reading and critique of the manuscript. Their suggestions have greatly improved the quality of the paper.

\section{Author details}

Vietnam National University, Hanoi, Hanoi, Vietnam. ${ }^{2}$ University of Technology, Sydney, Sydney, New South Wales, Australia.

Received: 8 January 2013 Accepted: 12 August 2013

Published: 3 September 2013

\section{References}

1. IEEE, IEEE Std 802, Air interface for fixed and mobile broadband wireless access systems, Amendment 2: physical and medium access control layer for combined fixed and mobile operation in licensed bands corrigendum 1 (IEEE, 2005). http:/ ieeexplore.eee.org/serlet/opac?punumber=10676. Accessed 13 September 2006

2. HS Wang, N Moayeri, Finite-state Markov channel - a useful model for radio communication channels. IEEE Trans Veh Technol 44(1), 163-171 (1995)

3. Q Zhang, SA Kassam, Finite-state Markov model for Rayleigh fading channels. IEEE Trans Commun 47(11), 1688-1692 (1999)

4. AJ Goldsmith, SG Chua, Variable-rate variable-power MQAM for fading channels. IEEE Trans Commun 45(10), 1218-1230 (1997)

5. AJ Goldsmith, SG Chua, Adaptive coded modulation for fading channels. IEEE Trans Commun 46(5), 595-602 (1998)

6. D-T Nguyen, V Xuan-Thang, N Quoc-Tuan, An FSMC model for the ACM scheme with repetition coding in mobile WiMAX, in Proceedings of the international conference on advanced technology for communications ATC'OS (, Hai Phong, Vietnam, 2009)

7. M Di Renzo, F Graziosi, F Santucci, Further results on the approximation of log-normal power sum via Pearson type IV distribution: a general formula for log-moments computation. IEEE Trans Commun 57(4), 893-898 (2009)

8. M Di Renzo, F Graziosi, F Santucci, A general formula for log-MGF computation: application to the approximation of log-normal power sum via Pearson type IV distribution, in Proceedings of IEEE vehicular technology conference, Singapore, 11-14 May (IEEE, New York, 2008), pp. 999-1003

9. NB Mehta, Approximating a sum of random variables with a lognormal. IEEE Trans Wire Commun 6(7), 2690-2699 (2007)

10. J Salo, A sum-product model as a physical basis for shadow fading. arXiv:cs/ 0702098v, 2007. Accessed 21 November 2011

11. H Suzuki, A statistical model for urban radio propagation. IEEE Trans Commun 25(7), 673-680 (1977)

12. JG Proakis, Digital Communications, 3rd edn. (McGraw-Hill, New York, 1995)

13. M Abramovitz, IA Stegun, Handbook of Mathematical Functions with Formulas, Graphs, and Mathematical Tables, 9th edn. (Dover, New York, 1972)
14. M Gudmundson, A correlation model for shadow fading in mobile radio. Electron Lett 27, 2146-2147 (1991)

15. A Conti, MZ Win, M Chiani, Slow adaptive M-QAM with diversity in fast fading and shadowing. IEEE Trans Commun 55(5), 895-905 (2007)

16. IS Gradshteyn, IM Ryzhik, Table of Integrals Series and Products (Academic Press, New York and London, 1965)

doi:10.1186/1687-1499-2013-219

Cite this article as: Quoc-Tuan et al: A 10-state model for an AMC scheme with repetition coding in mobile wireless networks. EURASIP Journal on Wireless Communications and Networking 2013 2013:219.

\section{Submit your manuscript to a SpringerOpen ${ }^{\odot}$ journal and benefit from:}

- Convenient online submission

- Rigorous peer review

- Immediate publication on acceptance

- Open access: articles freely available online

- High visibility within the field

- Retaining the copyright to your article

Submit your next manuscript at $\gg$ springeropen.com 